\title{
Detecting low-dimensional chaos by the "noise titration" technique: Possible problems and remedies
}

\author{
Jianbo Gao ${ }^{\mathrm{a}, \mathrm{b}, *}$, Jing $\mathrm{Hu}^{\mathrm{c}}$, Xiang Mao ${ }^{\mathrm{d}}$, Wen-wen Tung ${ }^{\mathrm{e}}$ \\ a PMB Intelligence LLC, PO Box 2077, West Lafayette, IN 47996, USA \\ ${ }^{\mathrm{b}}$ LNM, Institute of Mechanics, Chinese Academy of Sciences, Beijing, People's Republic of China \\ ${ }^{\mathrm{c}}$ Affymetrix, Inc., 3380 Central Expressway, Santa Clara, CA 95051, USA \\ ${ }^{\mathrm{d}}$ Department of Electrical and Computer Engineering, University of Florida, Gainesville, FL 32611, USA \\ e Department of Earth and Atmospheric Sciences, Purdue University, West Lafayette, IN 47907, USA
}

\section{A R T I C L E I N F O}

\section{Article history:}

Received 17 January 2011

Accepted 5 December 2011

Available online 20 January 2012

\begin{abstract}
A B S T R A C T
Distinguishing low-dimensional chaos from noise is an important issue in time series analysis. Among the many methods proposed for this purpose is the noise titration technique, which quantifies the amount of noise that needs to be added to the signal to fully destroy its nonlinearity. Two groups of researchers recently have questioned the validity of the technique. In this paper, we report a broad range of situations where the noise titration technique fails, and offer solutions to fix the problems identified.
\end{abstract}

(c) 2011 Elsevier Ltd. All rights reserved.

\section{Introduction}

Detecting low-dimensional chaos from noisy time series is a classic issue with tremendous importance in as diverse fields as life sciences, finance, ecology, physics, fluid mechanics, and geophysics. Two major difficulties for solving this issue are (i) chaos can be induced by noise [1-8], and (ii) standard Brownian motions may have a deterministic origin $[8,9]$. Although many methods have been proposed to distinguish chaos from noise [9-27], it is generally difficult to fully sort out the capabilities and limitations of a particular method.

Recently, an interesting method, called noise titration technique, which was introduced in 1996 for detecting nonlinearity [25], and later for detecting chaos [26], has been scrutinized $[28,29]$. The technique consists of detecting nonlinearity in a time series through Volterra series expansion and gradually adding noise to the signal till the nonlinearity is fully destroyed [25]. As a measure for chaos, a quantity, called noise limit $(N L)$, is introduced [26]. It is defined as $N L=100 \sigma_{n} / \sigma_{s}$, where $\sigma_{s}$ is the standard

\footnotetext{
* Corresponding author at: PMB Intelligence LLC, PO Box 2077, West Lafayette, IN 47996, USA.

E-mail address: jbgao@pmbintelligence.com (J. Gao).
}

deviation of the signal and $\sigma_{n}$ is the standard deviation of the minimal amount of noise that needs to be added to the signal to fully destroy its nonlinearity. This approach has been instrumental in inferring chaos in cardiac dynamics [30] and atomic force microscopy [31].

Two groups of researchers recently have questioned the validity of this noise titration technique by using nonlinear discrete maps with dynamical noise as counter-examples $[28,29]$. It is well-known that in nonlinear dynamical systems, dynamical noise can induce a number of interesting phenomena, including stochastic resonance [32] (for a review, see [33]), noise-induced instability [34,35], noiseinduced order [36], noise-induced multistability [37], and noise-induced chaos [1-8]. The last is the most relevant to the recent criticisms on the noise titration technique, as one can ask: are the dynamics of the noisy maps considered in $[28,29]$ simply stochastic or belong to noise-induced chaos? Unfortunately, neither group of researchers has considered this important issue. Consequentially, their criticisms remain inconclusive. More importantly, it is unclear when the noise titration technique may fail.

Given a finite time series, one only observes a finite scale range, $\left(\varepsilon_{\min }, \varepsilon_{\max }\right)[38,39]$. In a noisy dynamical system, when chaos (including noise-induced chaos) occurs, 
its signatures usually can only be observed in a subset of this scale range, say $\left(\varepsilon_{1}, \varepsilon_{2}\right)$, where $\varepsilon_{\min }<\varepsilon_{1}<\varepsilon_{2}<\varepsilon_{\max }$. On scales smaller than $\varepsilon_{1}$, noise dominates, while on scales larger than $\varepsilon_{2}$, fine structures of chaos cannot be resolved. If the scale spread $r=\varepsilon_{1} / \varepsilon_{2}$ is very close to 1 , then it is not meaningful to classify the motion as chaos [8-11]. Therefore, when low-dimensional chaos is concerned, one has to require $r \geqslant r^{*}>1$, where $r^{*}$ is a threshold of the scale spread. Note that in order to detect such scale-dependent chaotic signatures, one has no choice but to use a scaledependent complexity measure, since the task is not only to determine whether the motion is chaotic or not, but also to specify the scale range where chaos is observed.

There is no golden rule to choose $r^{*}$. The strategy advocated by Cencini et al. [8] is to renounce from the outset to define the nature of the signal and only speak about its character on a given range of scales according to certain scale-dependent indicators in that range of scales. Considering that some chaotic systems may have very small positive Lyapunov exponent and that the dimension of an actual signal may not be very small, here, we choose $r^{*}=2$, meaning that the scale spread is at least twofold. While our choice of $r^{*}$ is somewhat arbitrary and very non-conservative, it will suffice to serve our purpose of elucidating the problems with the noise titration technique.

There exist three scale-dependent complexity measures for tackling the above task. One is the $\varepsilon-\tau$ entropy [40]. Another is the finite size Lyapunov exponent (FSLE) $[8,41,42]$. The third is the scale-dependent Lyapunov exponent (SDLE) [9-12]. Conceptually, FSLE and SDLE are closely related. However, there are important differences between them, one being that FSLE cannot deal with systems with a negative largest Lyapunov exponent (LLE), while SDLE can. Since one of the models studied by Freitas et al. [28] has a negative LLE, therefore, FSLE is not suitable here. Computationally, $\varepsilon-\tau$ entropy is the least convenient, while SDLE is the most convenient. Therefore, in this study, we use SDLE to determine whether the recent criticisms on the noise titration technique are legitimate, and more importantly, to find solutions to the problems identified.

The remainder of the paper is organized as follows. In Section 2, we first overview the known properties of SDLE, then further explore the capabilities of SDLE in detecting chaos by studying high-dimensional and intermittent chaos. In Section 3, by studying both discrete- and continuous-time dynamical systems, we identify a broad range of situations where the noise titration technique may fail in reliably detecting chaos. In Section 4, we make a few concluding remarks.

\section{Detecting chaos by SDLE}

\subsection{SDLE as a multiscale complexity measure}

SDLE is defined in a phase space through consideration of an ensemble of trajectories [9-11]. In the case of a scalar time series $x(1), x(2), \ldots, x(n)$, a suitable phase space may be obtained by using time delay embedding [43-45] to construct vectors of the form:
$V_{i}=[x(i), x(i+L), \ldots, x(i+(m-1) L)]$,

where $m$ and $L$ are called the embedding dimension and the delay time, respectively. For chaotic systems, $m$ and $L$ have to be chosen according to certain optimization criterion $[10,21,38,46-50]$. For a stochastic process, which is infinite-dimensional, the embedding procedure transforms a self-affine stochastic process to a self-similar process in a phase space, and often $m=2$ is not only sufficient but also best illustrates a non-chaotic scaling behavior from a finite dataset [9-11].

SDLE is defined as follows. Denote the initial distance between two nearby trajectories by $\varepsilon_{0}$, and their average distances at time $t$ and $t+\Delta t$ respectively, by $\varepsilon_{t}$ and $\varepsilon_{t+\Delta t}$, where $\Delta t$ is small. Being defined in an average sense, $\varepsilon_{t}$ and $\varepsilon_{t+\Delta t}$ can be readily computed from any processes, even if they are non-differentiable stochastic processes. The SDLE $\lambda\left(\varepsilon_{t}\right)$ is defined by [9-11]

$\varepsilon_{t+\Delta t}=\varepsilon_{t} e^{\lambda\left(\varepsilon_{t}\right) \Delta t}, \quad$ or $\quad \lambda\left(\varepsilon_{t}\right)=\frac{\ln \varepsilon_{t+\Delta t}-\ln \varepsilon_{t}}{\Delta t}$.

Or equivalently by,

$\frac{d \varepsilon_{t}}{d t}=\lambda\left(\varepsilon_{t}\right) \varepsilon_{t}, \quad$ or $\quad \frac{d \ln \varepsilon_{t}}{d t}=\lambda\left(\varepsilon_{t}\right)$

To compute SDLE, we can start from an arbitrary number of shells,

$\varepsilon_{k} \leqslant\left\|V_{i}-V_{j}\right\| \leqslant \varepsilon_{k}+\Delta \varepsilon_{k}, \quad k=1,2,3, \ldots$,

where $V_{i}, V_{j}$ are reconstructed vectors, $\varepsilon_{k}$ (the radius of the shell) and $\Delta \varepsilon_{k}$ (the width of the shell) are arbitrarily chosen small distances ( $\Delta \varepsilon_{k}$ is not necessarily a constant). Then we monitor the evolution of all pairs of points $\left(V_{i}, V_{j}\right)$ within a shell and take average. Assuming that the order of averaging and taking logarithm in Eq. (2) can be interchanged, Eq. (2) can now be written as

$\lambda\left(\varepsilon_{t}\right)=\frac{\left\langle\ln \left\|V_{i+t+\Delta t}-V_{j+t+\Delta t}\right\|-\ln \left\|V_{i+t}-V_{j+t}\right\|\right\rangle}{\Delta t}$,

where $t$ and $\Delta t$ are integers in unit of the sampling time, and the angle brackets denote average within a shell.

Note that the initial set of shells for computing SDLE serve as initial values of the scales; through evolution of the dynamics, they will automatically converge to the range of inherent scales-which are the scales that define Eqs. (2) and (3). Also note that when analyzing chaotic time series, the condition

$|j-i| \geqslant(m-1) L$

needs to be imposed when finding pairs of vectors within a shell, to eliminate the effects of tangential motions [21], and for an initial scale to converge to the inherent scales [10].

To better understand the notion of "inherent scales", it is beneficial to discuss the notion "characteristic scale" (or "limiting scale"), $\varepsilon_{\infty}$, defined as the scale where SDLE is close to 0 . It is closely related to the total variation or the energy of the signal (for example, for a chaotic system, $\varepsilon_{\infty}$ defines the size of the chaotic attractor). If one starts from $\varepsilon_{0} \ll \varepsilon_{\infty}$, then, regardless of whether the data is deterministically chaotic or simply random, $\varepsilon_{t}$ will initially increase 
with time and gradually settle around $\varepsilon_{\infty}$. Consequentially, $\lambda\left(\varepsilon_{t}\right)$ will be positive before $\varepsilon_{t}$ reaches $\varepsilon_{\infty}$. On the other hand, if one starts from $\varepsilon_{0} \gg \varepsilon_{\infty}$, then $\varepsilon_{t}$ will simply decrease, yielding negative $\lambda\left(\varepsilon_{t}\right)$, again regardless of whether the data are chaotic or random. When $\varepsilon_{0} \sim \varepsilon_{\infty}$, then $\lambda\left(\varepsilon_{t}\right)$ will stay around $0-$ note however, that $\varepsilon_{\infty}$ may not be a single point, but a function of time, such as a periodic function of time. These discussions make it clear that chaos can only be observed on scales much smaller than $\varepsilon_{\infty}$.

To better understand SDLE, we now point out a relation between SDLE and the positive LLE $\lambda_{1}$ estimated for a true chaotic signal using, say, the Wolf et al.'s algorithm [14]. It is given by [10]

$\lambda_{1}=\int_{0}^{\varepsilon^{*}} \lambda(\varepsilon) p(\varepsilon) d \varepsilon$,

where $\varepsilon^{*}$ is a scale parameter (for example, used for re-normalization when using Wolf et al.'s algorithm [14]), $p(\varepsilon)$ is the probability density function for the scale $\varepsilon$ given by

$p(\varepsilon)=Z \frac{d C(\varepsilon)}{d \varepsilon}$,

where $Z$ is a normalization constant satisfying $\int_{0}^{\varepsilon^{*}} p(\varepsilon)$ $d \varepsilon=1$, and $C(\varepsilon)$ is the well-known Grassberger-Procaccia's correlation integral [13]. Note that the lower-bound for the integration is set to be zero here. In practice, on scales smaller than $\varepsilon_{\min }$, the probability $p(\varepsilon)$ will be zero. Therefore, one could replace the lower-bound for the integration by $\varepsilon_{\text {min }}$.

We now list three interesting scaling laws of SDLE that are most relevant to this study:

1. For clean chaos on small scales, and noisy chaos with weak noise on intermediate scales,

$$
\lambda(\varepsilon)=\lambda_{1} \text {. }
$$

Following our general discussion in the Introduction, we now explicitly define low-dimensional chaos to be observing scaling of Eq. (9) on a scale range of $(\varepsilon, r \varepsilon)$, where $r \geqslant 2$. As we will see in Section 2.2, such a definition of chaos is able to detect chaos in intermittent time series with a long laminar phase during which neighboring trajectories do not diverge, and a rapid divergence over a small part of the state space, as well as chaos from time series with multiple positive Lyapunov exponents and very high dimension (say, more than 20). However, it should be noted that when the dimension of a signal is very high, the scale range for observing Eq. (9) could be very narrow.

2. For clean chaos on large scales where memory has been lost and for noisy chaos (including chaos with measurement/dynamical noise and noise-induced chaos [6,7]) on small scales,

$$
\lambda(\varepsilon) \sim-\gamma \ln \varepsilon
$$

where $\gamma>0$ is a parameter. Note for noisy systems, on smallest scales, this is the only scaling-more data only resolves this scaling better. In the case of infinite data, one can imagine that this scaling extends to $\varepsilon \rightarrow 0$, meaning entropy is infinite in noisy systems. Also note that even for clean chaotic systems, this scaling would be observed on the smallest scales [38,39], if one does not impose the condition expressed by inequality (6).

3. For random $1 / f^{2 H+1}$ processes, where $0<H<1$ is called the Hurst parameter which characterizes the correlation structure of the process: depending on whether $H$ is smaller than, equal to, or larger than $1 / 2$, the process is said to have anti-persistent, short-range, or persistent long-range correlations $[51,10]$,

$$
\lambda(\varepsilon) \sim \varepsilon^{-1 / H}
$$

Note that for white Gaussian noise (whose integration is the standard Brownian motion), $H=1 / 2$, while for turbulence, $H=1 / 3$.

Before we use an example to illustrate the above scaling laws, it is important to point out how an estimated positive LLE cannot be used to indicate existence of low-dimensional chaos. For this purpose, let us consider white noise. Let $\bar{D}$ be the average distance between two points. If one chooses two points whose distance is smaller than $\bar{D}$, then the distance between them will rapidly grow with time, till their distance is close to $\bar{D}$. This means "LLE", as obtained by, say, the Wolf et al.'s algorithm [14], is positive. Obviously, associating such positive LLE with chaos is incorrect, since we are talking about simple noise. In the case of more complex noisy processes, if one evaluates Eq. (7) using Eqs. (10) and (11) for SDLE, then one gets positive LLE. These discussions make it clear that a positive LLE alone cannot be used as a test for low-dimensional chaos.

To better understand the above discussions, let us say a few more words about Eq. (7). What distinguishes Eq. (9) from (10) or (11) is the fact that when $\varepsilon^{*}$ in Eq. (7) changes, $\lambda_{1}$ also changes when one uses Eq. (10) or (11). However, so long as $\varepsilon^{*}$ lies in the plateau region, $\lambda_{1}$ is a fixed value when one evaluates Eq. (7) using Eq. (9). Noting that different researchers can choose different $\varepsilon^{*}$, the above discussions simply translate to that Eq. (10) or (11) characterizes random behavior, since LLE obtained by different researchers can be different, while Eq. (9) characterizes deterministic behavior.

We now illustrate the above scaling laws by using the following Lorenz system with dynamical noise as an example:

$d x / d t=-10(x-y)+D \eta_{1}(t)$

$d y / d t=-x z+28 x-y+D \eta_{2}(t)$,

$d z / d t=x y-\frac{8}{3} z+D \eta_{3}(t)$

where $D \eta_{i}(t), i=1,2,3$ are independent Gaussian noise forcing terms with mean 0 and variance $D^{2}$. Note that the system with other parameter values and measurement noise was studied earlier [9-11]. The system is solved using the scheme of Exact propagator [52], which involves solving the deterministic part of the system using a 4-th order Runge-Kutta method with a time-step of $h=0.002$, then adding a term $D \sqrt{h} W$, where $W$ is a Gaussian noise of mean 0 and variance 1 , to the corresponding equations to take into account the effect of noise. In order to compute SDLE, time series of length 10,000 , sampled with a time interval of 0.06 , is used, together with $m=4, L=2$. Fig. 1(a) shows five curves, for the cases of $D=0,1,2,3$, 

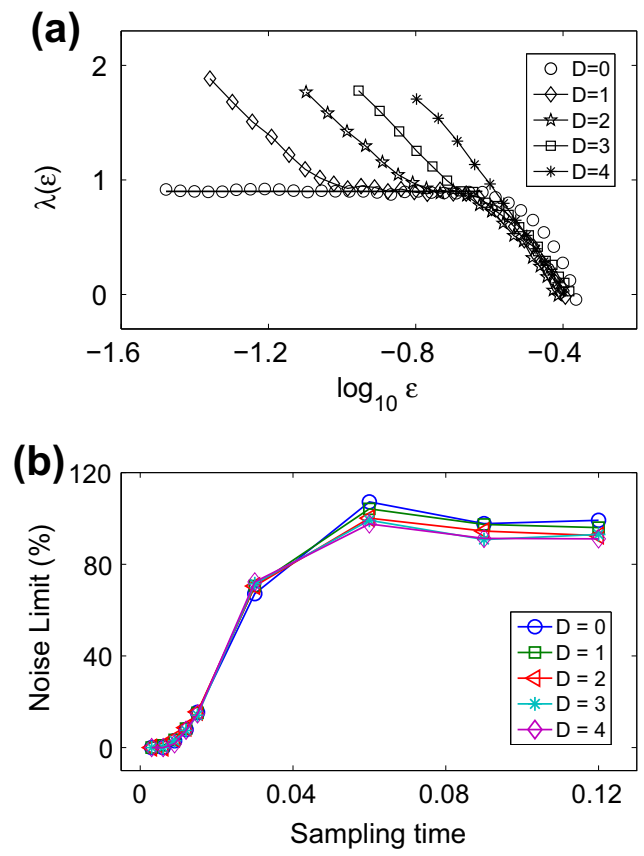

Fig. 1. (a) $\lambda(\varepsilon)$ curves for the clean and the noisy Lorenz system, data of length 10,000 points and embedding parameters $m=4, L=2$ were used for calculating SDLE curves. The shell sizes are $\left(2^{-(i+1) / 2}, 2^{-i}\right)$, $i=10,11, \ldots, 18$. (b) Variation of the noise limit with the sampling time for the Lorenz system with different noise levels.

4. We observe a few interesting features: (i) For the clean chaotic signal, on small scales, $\lambda(\varepsilon)$ slightly fluctuates around 0.9 , which is the LLE; on large scales where memory has been lost, we observe the scaling of Eq. (10). (ii) When there is stochastic forcing, $\lambda(\varepsilon)$ is no longer a constant when $\varepsilon$ is small, but increases as $-\gamma \ln \varepsilon$ when the scale $\varepsilon$ decreases. The coefficient $\gamma$ does not seem to depend on the strength of the noise. This feature suggests that entropy generation is infinite when the scale $\varepsilon$ approaches to zero. (iii) When the noise is increased, the part of the curve with $\lambda(\varepsilon) \sim-\gamma \ln \varepsilon$ shifts to the right, while the plateau defined by Eq. (9) shrinks. In fact, little chaotic signature can be identified when $D$ is increased beyond 3 . This simply means that the system has become too noisy to be classified as chaotic. However, we will show in Section 3 that the noise titration technique will still mis-classify such dynamics as chaos.

\subsection{Detecting high-dimensional and intermittent chaos}

In this subsection, we examine two model systems, one exhibits fairly high dimensions, the other exhibits intermittent chaos. We shall show that SDLE can readily detect chaos even in such systems.

Our first system is the Lorenz'96 model [53-55]. The model is supposed to represent some atmospheric quantity equally spaced around a latitudinal circle. It is described by the following equations,

$d X_{n} / d t=\left(X_{n+1}-X_{n-2}\right) X_{n-1}-X_{n}+F, \quad n=1,2, \ldots, N$ with periodic boundary condition, i.e., $X_{-1}=X_{N-1}, X_{0}=X_{N}$, and $X_{N+1}=X_{1} . F$ is a positive constant representing external forcing. The term $-X_{n}$ represents dissipation, and the term $\left(X_{n+1}-X_{n-2}\right) X_{n-1}$ represents advection. When $N=40$, $F=8$, the system has 13 positive Lyapunov exponents and a Kaplan-Yorke dimension of 27.1. In ensemble forecasting of this system, one chooses many points in a small neighborhood of a reference point, integrates the system starting from all those points, and monitors divergence between all those perturbed trajectories and the reference trajectory. The average of this divergence yields the root mean square error, which is also called the error growth curve. Since the system is chaotic, initially the error curve grows exponentially, or linearly in a semi-log plot, as shown by the solid blue curve in Fig. 2(a). Although the dimension here is very high, the SDLE shown in Fig. 2(b) still clearly indicate deterministic chaos, although the scale range for observing $\lambda(\varepsilon) \sim$ constant is fairly narrow. Note that integration of SDLE gives part of the error growth curve, as shown by the red solid curve in Fig. 2(a). Upon linear extrapolation of the solid red curve, one obtains the dashed red curve shown in Fig. 2(a), and recovers the entire error growth curve.

Next, we consider intermittent chaos. To illustrate the idea, we examine the logistic map

$x_{n+1}=a x_{n}\left(1-x_{n}\right)+\eta_{n}$.

Here, $\eta_{n}$ is a white Gaussian noise with mean 0 and standard deviation $\sigma$. It is a dynamical noise, since it is in the governing equation, and thus directly affects the evolution of the system (in contrast, $n(t)$ in $x(t)+n(t)$ is called measurement noise, since it only affects the value of $x(t)$, but not $x(t+1)$ ). When $\sigma=0$ and $a=3.8284$, we have intermittent chaos. An example of the time series is shown in Fig. 3(a). We observe that time intervals exhibiting chaos are very short compared with those exhibiting periodic motions. Traditional methods for computing Lyapunov exponent, being based on global average, is unable to quantify chaos in such an intermittent situation, since the laminar phase dominates. Neither can FSLE, since it requires that divergence dominates most of the time. Interestingly, the SDLE curve shown in Fig. 3(b) clearly indicates existence of chaotic motions, since the plateau region extends almost one order of magnitude.

Why can SDLE even detect chaos in such a situation? The reason is that the oscillatory part of the data only affects the scale range where $\lambda(\varepsilon) \sim 0$. It cannot affect the positive portion of $\lambda(\varepsilon)$. This means SDLE has a nice scale separation property to automatically separate the regular from chaotic motions.

Before leaving this example, we note that the $\ln \varepsilon$ scaling on very small scales is due to the transitions from periodic to chaotic motions. To understand this, consider two very close trajectories in the laminar region. So far as they stay in the laminar region, $\varepsilon$ will remain small. When both trajectories enter the chaotic region, the distance between them will become greater-this divergence becomes stronger and stronger when the trajectories get deeper into the chaotic region, till it stabilizes at the plateau region, when it is fully within the chaotic tunnel. 

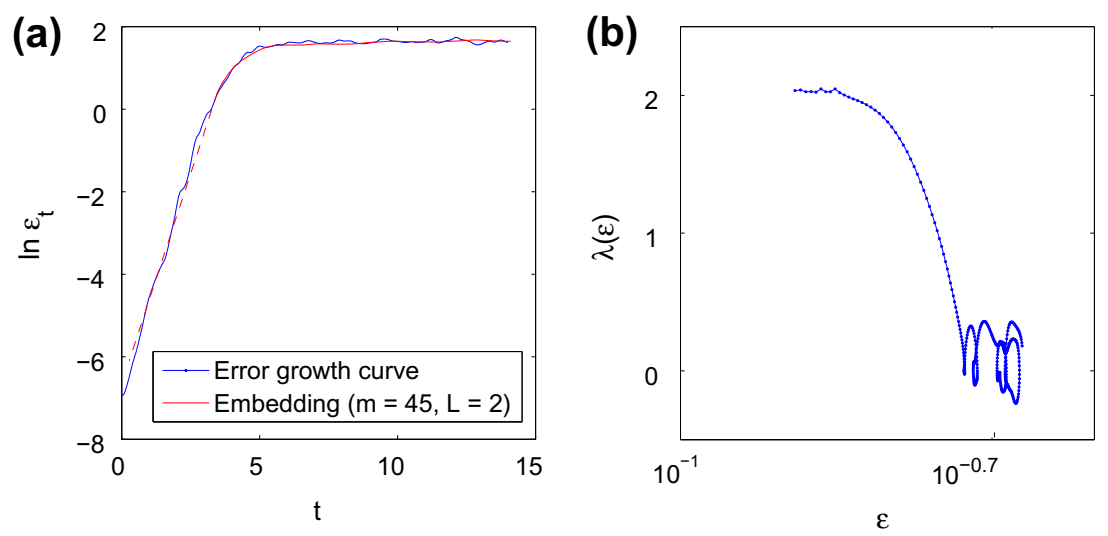

Fig. 2. (a) Error growth curves (in logarithmic scale) for the Lorenz ' 96 system. The blue curve is the average of the error curves of 250 ensembles, the red curve is the integration of SDLE (the dashed part of the red curve represents a linear extrapolation of the solid red curve). (b) $\lambda(\varepsilon)$ curve based on $X_{1}$ time series of the Lorenz ' 96 system. Data of length 480,000 points and embedding parameters $m=45, L=2$ were used for computing the SDLE. The curve shown in the figure corresponds to the shell with initial size $\left(2^{-5}, 2^{-4.5}\right)$. (For interpretation of the references to color in this figure legend, the reader is referred to the web version of this article.)
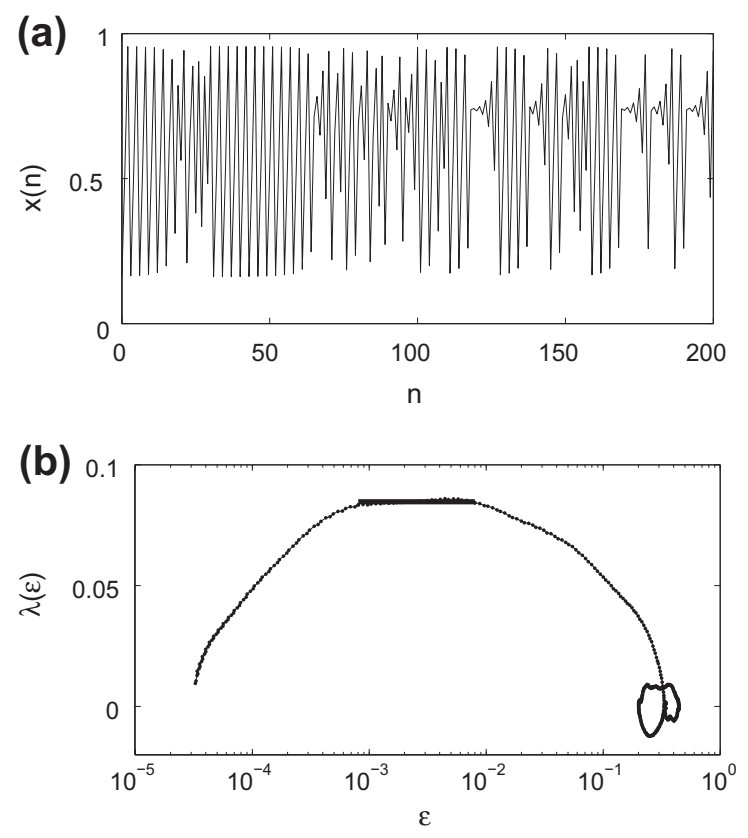

Fig. 3. (a) An intermittent time series generated by the logistic map with $a=3.8284$ and $\sigma=0$. (b) The SDLE curve for a time series of 10,000 points, with $m=4, L=1$, and a shell size of $\left(2^{-13.5}, 2^{-13}\right)$. A plateau is clearly visible.

\section{Detecting chaos by the noise titration technique}

To better appreciate the capabilities and limitations of the noise titration technique, in this section, we first overview some details of the technique, then study discrete and continuous dynamical systems, to identify a broad range of situations where the technique may fail.

\subsection{The noise titration technique}

The key element of the technique is the Volterra autoregressive representation:

$$
\begin{aligned}
x(n+1) & =k_{0}+\sum_{m_{1}=0}^{k-1} k_{1}\left(m_{1}\right) x\left(n-m_{1}\right) \\
& +\sum_{m_{1}=0}^{k-1} \sum_{m_{2}=0}^{k-1} k_{2}\left(m_{1}, m_{2}\right) x\left(n-m_{1}\right) x\left(n-m_{2}\right)+\cdots \\
& +\sum_{m_{1}=0}^{k-1} \cdots \sum_{m_{d}=0}^{k-1} k_{d}\left(m_{1}, m_{2}, \ldots, m_{d}\right) \prod_{i=1}^{d} x\left(n-m_{i}\right)
\end{aligned}
$$

where $x(n)$ is the time series, $k$ is the length of memory (which may be considered equivalent of the embedding dimension), $d$ is the order of the polynomial (when $d>1$, it is nonlinear), and $\left\{k_{r}\left(m_{1}, m_{2}, \ldots, m_{r}\right)\right\}$ are the coefficients of the Volterra series. This expansion creates $M=(k+d)$ !/ $k$ ! $d$ ! nonlinear models, with the order of polynomials ranging from 1 to $d$ and embedding dimension ranging from 1 to $k$. Such nonlinear models are then compared with linear models with order 1 and embedding dimension ranging from 1 to $M$, in terms of goodness of the fitting. The latter is quantified by the Akaike cost function, an information theoretic metric. If nonlinear models are better, then it is concluded that the time series is nonlinear and chaotic. To quantify chaos, noise is then added to the signal, till nonlinearity in the data can no longer be detected through such Volterra series expansion. Quantitatively, this is measured by noise limit, a metric we have explained in the Introduction.

Note that the technique does not involve characterization of any chaotic signatures of the time series. Instead, it tacitly assumes that when a nonlinear model better represents the data than a linear model, and that if the noise that needs to be added to the data to destroy nonlinearity is not zero, then the data is chaotic. Under such an assumption, clean, transient free periodic and quasi-periodic signals will be tested as linear and thus non-chaotic, even though such signals may actually be generated by nonlinear dynamical systems. Below, we shall identify a broad range of situations where problems with the noise titration technique may arise. 


\subsection{Detecting chaos in discrete dynamical systems using the} noise titration technique

In order to (1) identify a broader range of problems with the noise titration technique and (2) show how SDLE can fix the problems, in this subsection, we examine more systematically the three discrete maps studied in $[28,29]$. We first study the logistic map (Eq. (14)). Note that Freitas et al. [28] studied the clean map with $a=3.62$, while Lei and Meng [29] studied the noisy map with $a=3.3,3.84$, 3.9 and noise variance $\sigma^{2}=0.001$.

Fig. 4(a) shows the bifurcation diagram for the noisy logistic map with $\sigma=0.001$. We see that the major bifurcation structure of the clean logistic map can still be observed clearly. For each parameter $a$ and a fixed noise level, we can generate a time series and analyze it using the noise titration technique. The value of the noise limit vs. the parameter $a$ (mainly admitting period- 4 motions) is shown in Fig. 4(b). We observe that for $\sigma=0.001, N L$ is always positive for the entire interval of $a$ shown in the figure. Are those motions noise-induced chaos?

The answer is no. An example of SDLE for the case of $a=3.55$ is shown in Fig. 4(c). Clearly, there is no plateau defined by Eq. (9), therefore, it is not chaotic. For comparison purpose, we have shown in Fig. 4(d) the SDLE curve for the clean map with $a=3.62$, which is a chaotic case studied by Freitas et al. [28]. We have also shown in Fig. 4(e) the SDLE curve for the noisy map with $a=3.74$, which is a case of noise-induced chaos [6,9]. Note that the main feature of Fig. 4(c) remains the same when longer time series with larger embedding dimensions are used, such as $m \geqslant 20$.
To better understand why the time series of the noisy logistic map with $a=3.55$ cannot be classified as noiseinduced chaos, but rather a simple limit cycle driven by dynamical noise (i.e., a stochastic oscillator), it is instructive to compare its time series with that of a true noiseinduced chaos. For this purpose, we have shown in Fig. 5 a short segment of the time series for the logistic map with (a) $a=3.55, \sigma=0.01$ and (b) $a=3.74, \sigma=0.002$. We observe that for the case of $a=3.55$ and $\sigma=0.01$, the noisy time series is still very much like a period-4 oscillation-while the amplitude of the oscillation changes with time due to noise, the period is still precisely 4 . When $\sigma$ is 10 or 100 times smaller, as shown in Fig. 4(b), surely one would expect that the effect of noise is even smaller. However, this is not true in case(b). In fact, the noisy time series of $a=3.74$ bears no resemblance to the clean period- 5 oscillation of the clean system at all. This is the essence of noiseinduced chaos-even though noise is small, its effect is huge. Note however, the non-chaotic nature of the case of $a=3.55$ and $\sigma=0.01$ may not be inferred through examination of a bifurcation diagram, since the motion is not represented by 4 points in a bifurcation diagram, due to the amplitude variations. These discussions make it clear that there is no mechanism for exponential divergence in the case of $a=3.55$. Therefore, the motion of the noisy logistic map with $a=3.55$ cannot be chaotic.

Next, we consider how the chaotic motions of the clean logistic map are affected by dynamical noise. The behaviors are very much like those of the chaotic Lorenz system we already discussed, and therefore, will not be illustrated by any plots. We just want to emphasize that when noise is
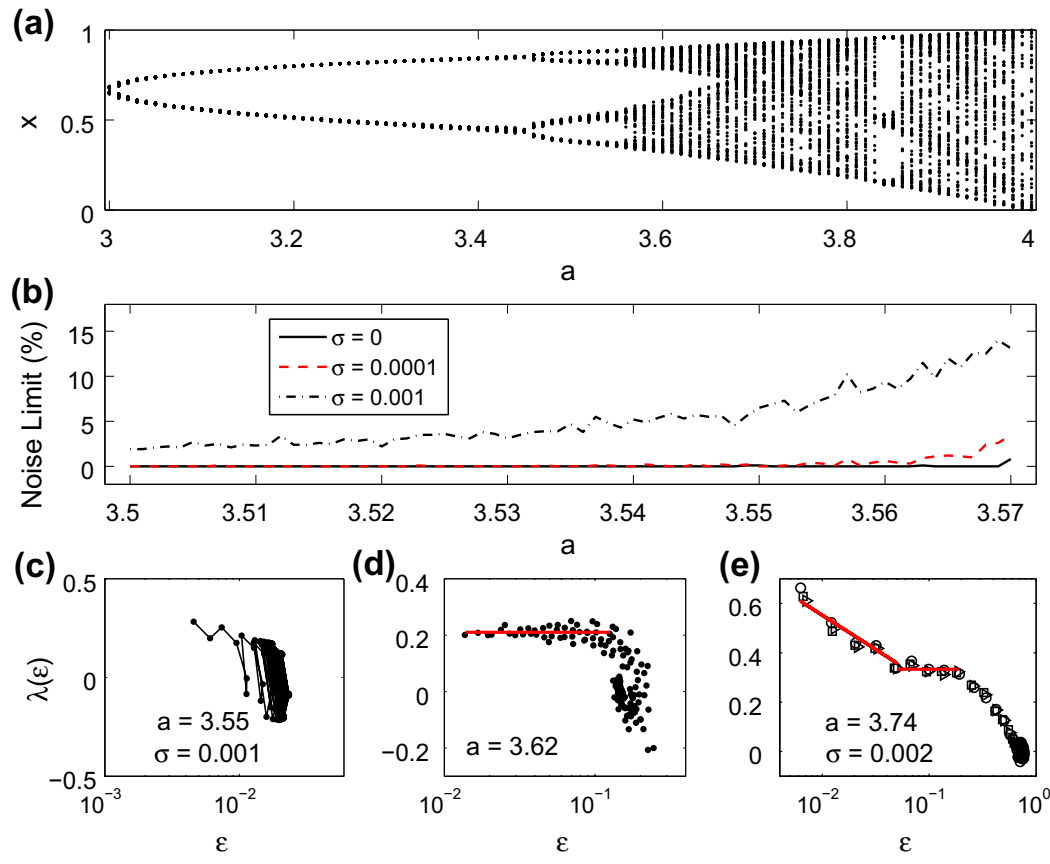

a
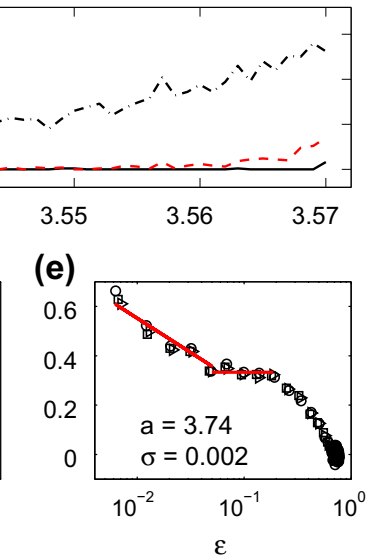

Fig. 4. (a) Bifurcation diagram of the noisy logistic map with $\sigma=0.0001$; (b) variation of $N L$ with the bifurcation parameter $a$ for three different noise levels; (c) $\lambda(\varepsilon)$ for the logistic map with $a=3.55, \sigma=0.001$, the curve was calculated based on a time series of 10,000 points, with $m=2, L=1$, and a shell size of $\left(2^{-11.5}, 2^{-11}\right)$; (d) $\lambda(\varepsilon)$ for the clean logistic map with $a=3.62$, the curves were calculated based on a time series of 20,000 points, with $m=2, L=1$, and correspond to 7 shells with size $\left(2^{-i-1 / 2}, 2^{-i}\right), i=4,4.5,5,5.5,6,6.5$, and 7 ; (e) $\lambda(\varepsilon)$ for the logistic map with $a=3.74, \sigma=0.0002$, the curves were calculated based on a time series of 20,000 points, with $m=4, L=1$, and correspond to 3 shells with size $\left(2^{-i-1 / 2}, 2^{-i}\right), i=9,9.5$, and 10 . 

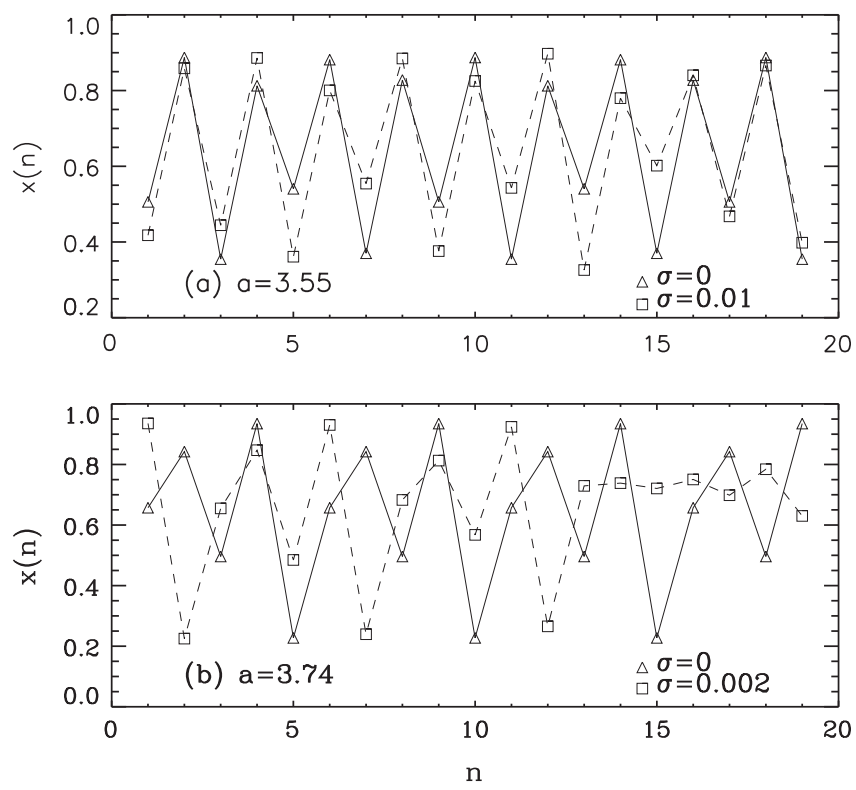

Fig. 5. Time series for the logistic map with (a) $a=3.55$ and (b) $a=3.74$. The points designated by open triangles and connected by solid curves are for the clean system, while the points designated by squares and connected by dashed lines are for the noisy system.

as large as that used by Lei and Meng [29], the time series for the case of $a=3.9$ is no longer chaotic, but simply stochastic. Therefore, the three cases, with $a=3.3,3.84$ and 3.9 , studied by Lei and Meng, which all yield $N L>0$, constitute three counter-examples of the noise titration technique (instead of two, as Lei and Meng originally thought).

Finally, we ask a question: May the noise titration technique even classify a non-chaotic deterministic time series as chaotic? To find the answer, we consider the clean logistic map at the accumulation point, $a_{\infty}=3.569945672 \cdots$. When $a$ is slightly smaller or larger than $a_{\infty}$, the motion is periodic or weakly chaotic, respectively [56]. The SDLE curve for the map at the accumulation point is shown in Fig. 6(b). Clearly it is not chaotic yet. However, the noise titration method yields a positive noise limit $(N L=0.41$ and 0.71 , for a time series of length 1000 and 10,000 , respectively). Note that when there is dynamical noise, irrespective of whether $a$ is slightly smaller than, equal to, or slightly larger than $a_{\infty}$, the motions are all characterized by power-law sensitivity to initial conditions (PSIC) [56], but not exponential sensitivity to initial conditions (ESIC). In all these cases, $N L$ are positive, with values ranging from slightly smaller than 3 to about 20, depending on the data length and the noise level.

We now summarize what we have found from the logistic map. We have found three types of problems with the noise titration technique: (1) the technique classifies a stochastic oscillation as chaos; (2) it also classifies a motion with its chaotic features completely destroyed by noise as chaos; and (3) it even classifies the motions near the accumulation point, which are either deterministic or characterized by power-law sensitivity to initial conditions, as chaos.

We now study the other two maps studied by Freitas et al. [28]. One is the sine map with a specially designed noise, as described below: $x_{n+1}=\mu \sin \left(x_{n}\right)+Y_{n} \eta_{n}$,

where $\mu=2.4, Y_{n}$ is a random variable from a Bernoulli process $\left(Y_{n}=1\right.$ or -1 with probabilities $q=0.01$ and $1-q=0.99$, respectively), and $\eta_{n}$ is an independently and identically distributed (iid) random variable with a uniform distribution between $(-b, b)$, where $b=2$. A noisy time series of the map is shown in Fig. 7(a). We observe that the motion of the map consists of two symmetric period-2 oscillations, and is occasionally disturbed by the noise. The first return map of the system is shown in Fig. 7(b). Note that the sine-like shape comes from the term $\mu$ sin $\left(x_{n}\right)$; the positions of $x_{n}$ where we observe four vertical lines are the positions of the period- 2 motions, while the four vertical lines are due to noise: had we used more points, they would have become more uniform, conforming to the uniform distributions used. Although this system yields a positive $N L$ value, as reported by Freitas et al. (and also verified by us), the SDLE plot shown in Fig. 7(c) does not suggest chaos at all. At this point, it is worth noting that the SDLE plot indicates a negative plateau, with average value about -0.6 , close to the negative LLE of -0.65 found by Freitas et al. [28]. However, the map, being disturbed by this specially designed noise, cannot be fully characterized by a negative LLE, since the pattern of the SDLE shown in Fig. 7(c) is quite complicated. Nevertheless, this is surely a valid counter example of the noise titration technique.

Finally, we examine model 2 studied by Freitas et al. [28], which is described by

$x_{n+1}=a v_{n}+b v_{n-1}\left(1-v_{n}\right)$,

where $v_{n}$ is a uniform iid random variable with values between 0 and 1 . The values of parameters $a$ and $b$ are not given in [28]. We have tried a number of their combinations. Here, for illustration purpose, we have chosen $a=3, b=4$, which yields a very similar phase diagram (i.e., the first return 

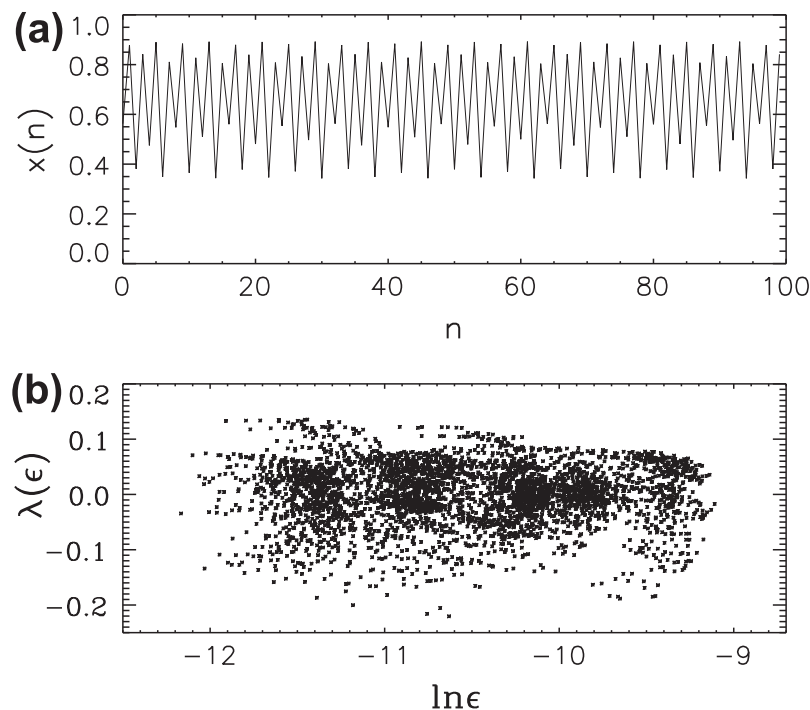

Fig. 6. (a) A short segment of the time series for the logistic map on the edge of chaos; (b) $\lambda(\varepsilon)$ for a data set of length 10,000 points, with $m=4, L=1$, and a shell size of $\left(2^{-17.5}, 2^{-17}\right)$.
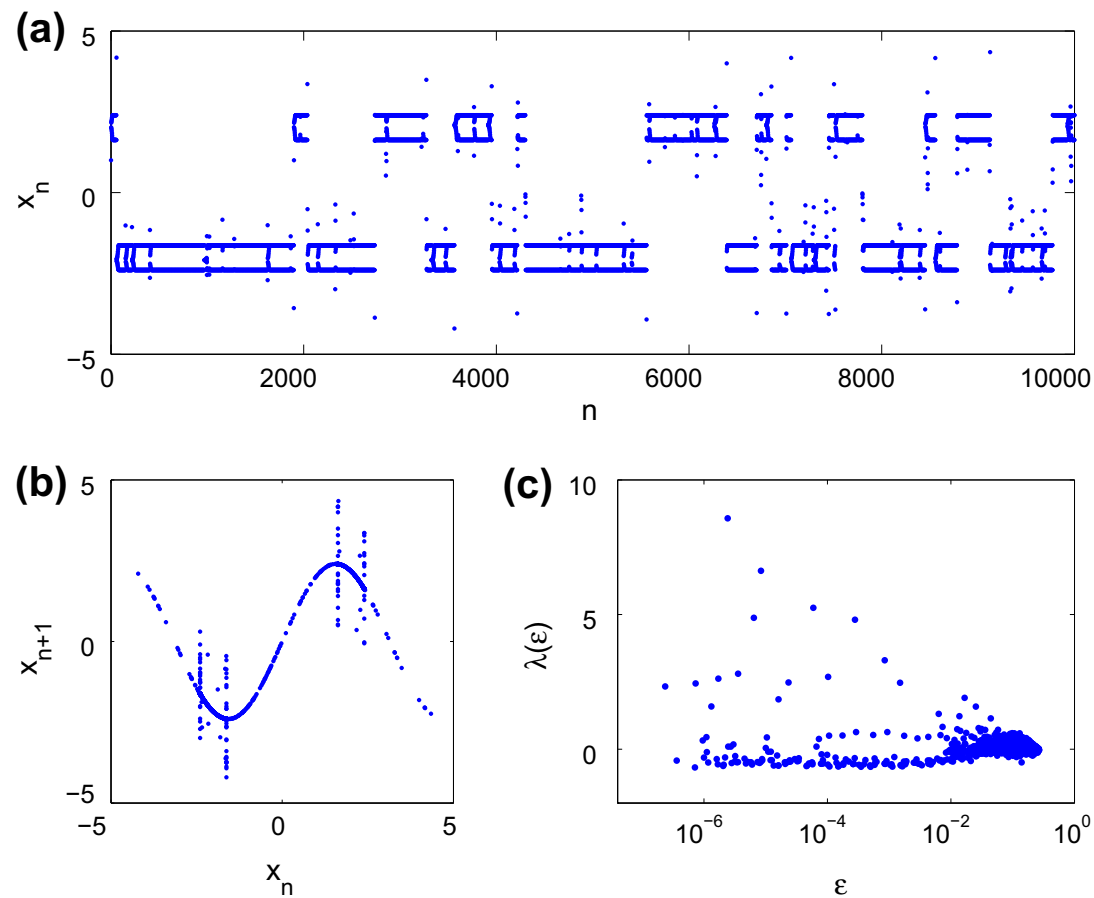

Fig. 7. (a) Time series generated by Eq. (16), (b) the first return map, and (c) the $\lambda(\varepsilon)$ curves calculated based on a time series of 10,000 points, with $m=2$, $L=1$, curves correspond to 7 shells with size $\left(2^{-i-1 / 2}, 2^{-i}\right), i=4,4.5,5,5.5,6,6.5$, and 7 .

map) to that shown in [28]. A short segment of the time series is shown in Fig. 8(a), while the SDLE curve is shown in Fig. 8(b). Clearly, the time series is not chaotic. However, the $N L$ is not only positive, but large, and sensitively depends on the data length, as shown in Fig. 8(c). Note that even though the parameters chosen here may not be identical to those used by Freitas et al., we can safely conclude that their result is correct, in the sense that the noise titration technique falsely classifies the stochastic motion as chaotic.
3.3. Detecting chaos in continuous dynamical systems using the noise titration technique

Many real world data are measured from continuous time systems. To better understand when the noise titration technique may fail, it is important to examine continuous time systems. Our analyses of discrete maps suggest that the more serious problems with the noise titration technique are to mis-classify stochastic oscillations and 

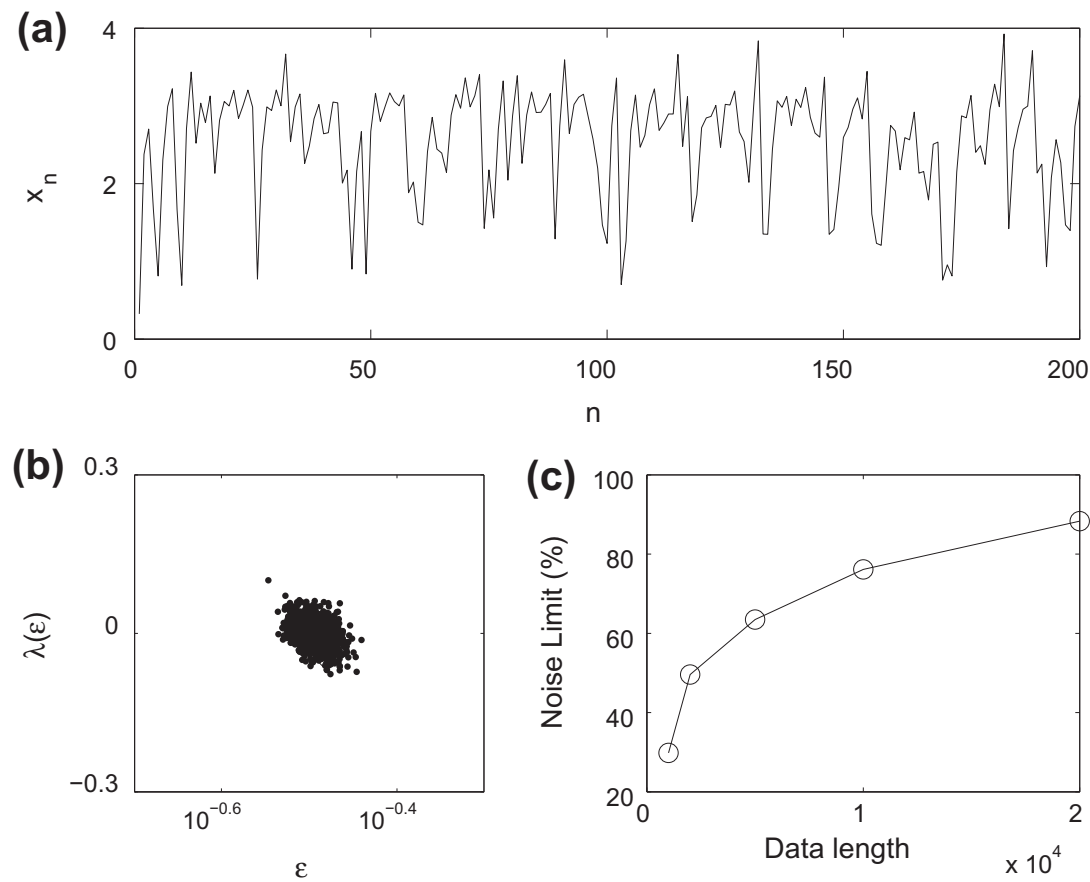

Fig. 8. (a) Time series generated by Eq. (17); (b) the $\lambda(\varepsilon)$ curve calculated based on a time series of 2000 points, with $m=2, L=1$, and a shell size of $\left(2^{-4}, 2^{-3.5}\right)$; and (c) the variation of the noise limit with data length.

stochastic motions without any chaotic signatures remaining as chaos. Therefore, here we focus on the stochastic van der Pol's oscillator and the noisy Lorenz system.

The stochastic van der Pol's oscillator is described by

$d x / d t=y+D_{1} \eta_{1}(t)$,

$d y / d t=-\left(x^{2}-1\right) y-x+D_{2} \eta_{2}(t)$

where $\left\langle\eta_{i}(t)\right\rangle=0,\left\langle\eta_{i}(t) \eta_{j}\left(t^{\prime}\right)\right\rangle=\delta_{i j} \delta\left(t-t^{\prime}\right), i, j=1,2$, and the parameters $D_{i}, i=1,2$ characterize the strength of noise. In [9], we studied the system with a very small noise, $D_{1}=D_{2}=0.02$. Here, we study the system with a much larger noise, $D_{1}=D_{2}=0.2$. Like the stochastic Lorenz system, it is solved using the scheme of Exact propagator [52]. Fig. 9(a) (dashed curve) shows a noisy time series sampled with a time interval of 0.2 . Its phase diagram, plotted in Fig. 9(b), shows clearly a diffused limit cycle. To better appreciate the regularity of the time series, we have also shown in Fig. 9(a) a time series for the clean van der Pol oscillator as the solid curve. While the effect of noise is clearly discernible, the regularity of the noisy data is also evident: the period of the oscillation remains fairly stable, just as we have observed with the noisy logistic map with $a=3.55$. Such behavior suggests that short-term prediction of such stochastic oscillations cannot be exact, because of noise; however, long-term prediction with certain accuracy is possible, since the period is well defined. This argument suggests that the motion cannot be classified as chaos. The validity of this conclusion can be readily verified by the SDLE curve shown in Fig. 9(c).

How will the noise titration technique classify such a motion? It yields a fairly large $N L$ of about 30 . In fact, if we downsample the data by a factor of $2,5,10$, or 20 , the value of $N L$ changes, as shown in Fig. 9(d). Therefore, the stochastic van der Pol oscillator is another counterexample of the noise titration technique. Not only so, our analysis has pointed to a further difficulty of the technique: the quantitative metric for chaos, the noise limit, varies with the sampling time. In practice, the last feature makes it difficult for different researchers to compare their results, since different researchers may choose different sampling times.

At this point, we wish to emphasize that we have applied the noise titration technique to some experimental data, including pathological tremors [57-59] and velocity signals measured in the near wake of a circular cylinder of low-Reynolds number [60]. Both types of data have been found not to be chaotic, but like stochastic oscillations. However, the noise titration method always classifies them as chaotic.

Finally, we re-examine the Lorenz system of Eq. (12) with different noise levels. We first recall that using SDLE, we have found that when the noise level $D$ increases, the plateau region where $\lambda(\varepsilon) \sim$ constant shrinks; in fact, when $D \geqslant 3$, little chaotic signature of the system can be found. How does the noise limit vary with the noise level? It turns out that irrespective of different noise level, the $N L$ values are always positive and similar, and vary with the sampling time, as shown in Fig. 1(b). This clearly indicates that the noise titration technique fails to distinguish true chaos from noise. At this point, we would like to point out one more complication in Fig. 1(b): when the sampling time is very small (such as 0.01 ), $N L=0$, suggesting non-chaotic dynamics even for the clean Lorenz system. As we already 

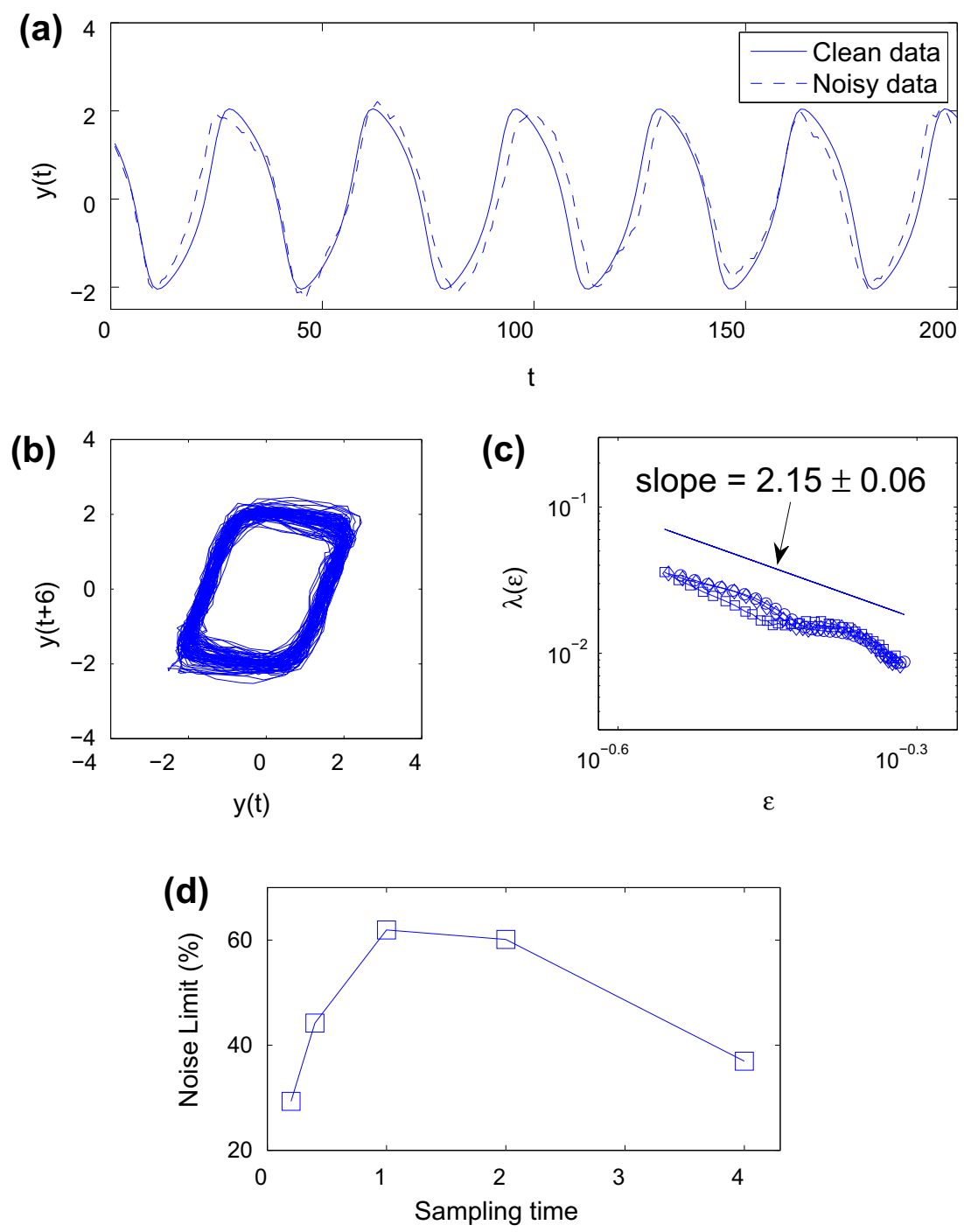

Fig. 9. (a) Time series of the clean and noisy van der Pol oscillator; (b) phase diagram for the noisy van der Pol oscillator; (c) $\lambda(\varepsilon)$ curves for the noisy oscillator showing power-law behavior, the curves were calculated based on a time series of 20,000 points, with $m=8, L=2$, and correspond to 3 shells with size $\left(2^{-i-1 / 2}, 2^{-i}\right), i=4,4.5$ and 5 ; (d) variation of the noise limit with the sampling time for the stochastic van der Pol oscillator.

commented earlier, the reason for such a behavior is that locally the signals can be perfectly linearized, and thus leading to misclassifying chaotic signals as non-chaotic.

In principle, are small sampling times allowed for a dynamical system? The answer is yes, since small sampling times do not violate the Nyquist sampling theorem at all. In fact, for the Lorenz system, when the sampling time is 0.01 , estimation of the correlation dimension and the largest Lyapunov exponent is as good as with larger sampling times $[61,62]$. Note that this issue is closely related to modeling problems. For an interesting review, we refer to [63].

\section{Concluding remarks}

By studying a number of discrete and continuous time systems, we have identified a broad range of problems with the noise titration technique, including two that could frequently occur in real applications: (1) stochastic oscillations and (2) chaotic systems with little chaotic signatures remaining due to excessive amount of noise.

What is the fundamental reason that the noise titration technique fails in so many situations? It is because of the assumption that nonlinearity implies chaos. Had a system to be studied is deterministic and dissipative, the noise titration technique could function very well, so far as the parameters of the system are not near the edge of chaos. This is because regular attractors of a deterministic dissipative system, including fixed point solutions, limit cycles, and torus, will not be detected as nonlinear, if transients have died out. The remaining major type of attractor, the chaotic attractor, will then be correctly classified as chaotic. However, whenever there is dynamical noise, both regular and chaotic attractors will be perturbed. Right after the perturbation, the process of converging to the original 
attractors will be initiated. In a dissipative system, while this process is necessarily nonlinear, there is no reason to classify them all as chaotic. This is the fundamental problem with the noise titration technique. Fortunately, all the problems identified can be readily solved by SDLE.

Detecting chaos from noisy time series has been and will remain as a fundamental issue in science and engineering. Therefore, there will be continuing efforts for developing new methods for this purpose. We wish to advocate that when one is making an effort to develop a new method, one better ensures that certain chaotic features of the data are characterized. Without such a requirement, problems are bound to occur.

\section{Acknowledgments}

The authors are grateful for Professor Leon Glass's suggestion of examining intermittent chaos and the 2nd model of Freitas et al., and two anonymous reviewers very constructive comments. This work is supported in part by NSF Grants CMMI-0825311 and 0826119.

\section{References}

[1] Crutchfield JP, Huberman BA. Phys Lett 1980;74A:407.

[2] Crutchfield JP, Farmer JD, Huberman BA. Phys Rep 1982;92:46.

[3] Kautz RL. J Appl Phys 1985;58:424.

[4] Arecchi F, Badii R, Politi A. Phys Rev A 1985;32:402.

[5] Gao JB, Tung WW, Rao N. Phys Rev Lett 2002;89:254101.

[6] Gao JB, Hwang SK, Liu JM. Phys Rev Lett 1999;82:1132-5; Gao JB, Chen CC, Hwang SK, Liu JM. Int J Mod Phys B 1999;13:3283-305.

[7] Hwang K, Gao JB, Liu JM. Phys Rev E 2000;61:5162-70.

[8] Cencini M, Falcioni M, Olbrich E, Kantz H, Vulpiani A. Phys Rev E 2000;62:427.

[9] Gao JB, Hu J, Tung W-W, Cao YH. Phys Rev E 2006;74:066204.

[10] Gao JB, Cao YH, Tung W-W, Hu J. Multiscale analysis of complex time series-integration of chaos and random fractal theory, and beyond. New York: Wiley Interscience; 2007.

[11] Hu J, Gao JB, Tung WW. Chaos (special issue on Controversial Topics in Nonlinear Science: Is the Normal Heart Rate Chaotic?) 2009;19:028506.

[12] Hu J, Gao JB, Tung WW, Cao YH. Multiscale analysis of heart rate variability: a comparison of different complexity measures. Ann Biomed Eng 2010;38:854-64.

[13] Grassberger P, Procaccia I. Phys Rev Lett 1983;50:346-9.

[14] Wolf A, Swift JB, Swinney HL, Vastano JA. Physica D 1985;16:285.

[15] Sugihara G, May RM. Nature 1990;344:734.

[16] Tsonis AA, Elsner JB. Nature 1992;358:217.

[17] Kaplan DT, Glass L. Phys Rev Lett 1992;68:427.

[18] Wayland R, Bromley D, Pickett D, Passamante A. Phys Rev Lett 1993;70:580.

[19] Salvino LMW, Cawley R. Phys Rev Lett 1994;73:1091.
[20] Pei X, Moss f. Nature 1996;379:618

[21] Gao JB, Zheng ZM. Phys Rev E 1994;49:3807-14.

[22] Gao JB, Zheng ZM. Europhys Lett 1994;25:485-90.

[23] Osborne AR, Provenzale A. Physica D 1989;35:357.

[24] Provenzale A, Osborne AR, Soj R. Physica D 1991;47:361.

[25] Barahona M, Poon CS. Nature 1996;381:215.

[26] Poon CS, Barahona M. Proc Natl Acad Sci USA 2001;98:7107-12.

[27] Hu J, Tung WW, Gao JB, Cao YH. Phys Rev E 2005;72:056207.

[28] Freitas US, Letellier C, Aguirre LA. Phys Rev E 2009;79:035201(R).

[29] Lei M, Meng G. Chaos Soliton Fract 2008;36:512.

[30] Wu GQ, Arzeno NM, Shen LL, Tang DK, Zheng DA, Zhao NQ, et al. PLoS ONE 2009;4:e4323.

[31] Hu SQ Raman A. Phys Rev Lett 2006;96:036107.

[32] Benzi R, Sutera A, Vulpiani A. J Phys A 1981;14:L453.

[33] Gammaitoni L, Hanggi P, Jung P, Marchesoni F. Rev Modern Phys $1998 ; 70: 223$

[34] Bulsara AR, Jacobs EW, Schieve WC. Phys Rev A 1990;42:4614.

[35] Chen ZY. Phys Rev A 1990;42:5837.

[36] Matsumoto K, Tsuda I. J Stat Phys 1983;31:87.

[37] Tung WW, Hu J, Gao JB, Billock VJ. Int J Bifurcat Chaos 2008;18:1749.

[38] Kantz H, Schreiber T. Nonlinear time series analysis. 2nd ed. Cambridge University Press; 2004.

[39] Olbrich E, Kantz H. Phys Lett A 1997;232:63-9.

[40] Gaspard P, Wang XJ. Phys Rep 1993;235:291-343.

[41] Aurell E, Boffetta G, Crisanti A, Paladin G, Vulpiani A. J Phys A 1997;30:1-26.

[42] Boffetta G, Cencini M, Falcioni M, Vulpiani A. Phys Rep 2002;356:367-474.

[43] Packard NH, Crutchfield JP, Farmer JD, Shaw RS. Geometry from a time series. Phys Rev Lett 1980;45:712-6.

[44] Takens F. Detecting strange attractors in turbulence. In: Rand DA, Young LS, editors. Dynamical systems and turbulence. Lecture notes in mathematics, vol. 898. Berlin: Springer-Verlag; 1981. p. 366.

[45] Sauer T, Yorke JA, Casdagli M. Embedology. J Stat Phys 1991;65:579-616

[46] Cenys A, Pyragas K. Phys Lett A 1988;129:227-30.

[47] Aleksic Z. Physica D 1991;52:362-8.

[48] Liebert W, Pawelzik K, Schuster HG. Europhys Lett 1991;14:521-6.

[49] Kennel MB, Brown R, Abarbanel HDI. Phys Rev A 1992;45:3403-11.

[50] Fraser AM, Swinney HL. Phys Rev A 1986;33:1134-40.

[51] Gao JB, Hu J, Tung WW, Cao YH, Sarshar N, Roychowdhury VP. Assessment of long range correlation in time series: how to avoid pitfalls. Phys Rev E 2006;73:016117.

[52] Mannella R. Int J Modern Phys C 2002;13:1177.

[53] Lorenz EN. J Atmos Sci 2005;62:1574-87.

[54] Lorenz EN. Proceedings of the seminar on predictability, vol. 1. Reading, Berkshire, United Kingdom: ECMWF; 1996.

[55] Lorenz EN, Emanuel KA. J Atmos Sci 1998;55:399.

[56] Gao JB, Tung WW, Cao YH, Hu J, Qi Y. Physica A 2005;353:613-24.

[57] Timmer J, Haussler S, Lauk M, Lucking CH. Chaos 2000;10:278288.

[58] Gao JB, Tung WW. Biol Cybernet 2002;86:263-70.

[59] Gao JB. Med Biol Eng Comput 2004;52:345-9.

[60] Gao JB. Physica D 1997;106:49.

[61] Gao JB, Sultan H, Hu J, Tung WW. Denoising nonlinear time series by adaptive filtering and wavelet shrinkage: a comparison. IEEE Signal Process Lett 2010;17:237-40.

[62] Tung WW, Gao JB, Hu J, Yang L. Recovering chaotic signals in heavy noise environments. Phys Rev E 2011;83:046210.

[63] Aguirre LA, Letellier C. Modeling nonlinear dynamics and chaos: a review. Math Probl Eng; 2009. doi:10.1155/2009/238960. 